\title{
Studies on the Total Synthesis of Lactonamycin:
}

\section{Synthesis of the CDEF Ring System}

Hermut Wehlan, ${ }^{\dagger}$ Eva Jezek, ${ }^{\dagger}$ Nathalie Lebrasseur, ${ }^{\dagger}$ Grégoire Pavé, ${ }^{\dagger}$ Emmanuel Roulland,${ }^{\dagger}$ Andrew J. P. White, ${ }^{\dagger}$ Jeremy N. Burrows ${ }^{\ddagger}$ and Anthony G. M. Barrett ${ }^{\dagger *}$

Department of Chemistry, Imperial College London, South Kensington, London SW7 2AZ, England and AstraZeneca, Sodertalje, S-151 85, Sweden.

\section{Supporting Information}

\section{Table of Contents}

General Procedures

Page S3

1-Bromomethyl-2,5-dimethoxy-3-(methoxymethyl)-benzene (7a).

Page S3

1-Bromomethyl-2,5-dimethoxy-3-((4-methoxybenzyloxy)methyl)-benzene (7b).

Page S4

Methyl 2-hydroxy-4,6-dimethoxybenzoate (10).

Page S4

Methyl 3-formyl-2-hydroxy-4,6-dimethoxybenzoate (11).

Page S5

Dimethyl 5-chloro-2-hydroxy-4,6-dimethoxy-1,3-benzenedicarboxylate (16).

Page S5

Methyl 5,7-dimethoxy-1,3-dioxo-2,3-dihydro-1H-isoindole-4-carboxylate (17).

Page S5

Methyl 3-ethoxy-5,7-dimethoxy-1-oxo-2,3-dihydro-1H-isoindole-4-carboxylate (18). $\quad$ Page S6

Methyl 5,7-dimethoxy-2-methyl-1,3-dioxo-2,3-dihydro-1H-isoindole-4-carboxylate (19a).

Page S6 
Methyl 7-methoxy-2-methyl-1,3-dioxo-5-trifluoromethanesulfonyloxy-2,3-dihydro- Page S6 $1 H$-isoindole-4-carboxylate (19b).

Methyl 5-(2,5-dimethoxybenzyl)-7-methoxy-2-methyl-1-oxo-2,3-dihydro-1Hisoindole-4-carboxylate $(\mathbf{2 1})$.

Page S7

5-(2,5-Dimethoxybenzyl)-7-methoxy-2-methyl-1-oxo-2,3-dihydro-1H-isoindole-4carboxylic acid (22).

Page S8

2-Methyl-4,7,10,11-tetramethoxy-1,2-dihydronaphtho[2,3-e]isoindol-3-one (24a). $\quad$ Page S8

2-Methyl-3-oxo-4,7,10-trimethoxy-2,3-dihydro-1H-naphtho[2,3-e]isoindol-11-yl Page S9 acetate $\mathbf{( 2 4 b )}$.

4-Methoxy-2-methyl-3,7,10-trioxo-2,3,7,10-tetrahydro-1H-naphtho[2,3-e]isoindol-11- Page S9 yl acetate $\mathbf{( 2 5 b )}$.

2-Hydroxymethyl-4-methoxy-6-(methoxymethyl)-phenol (28).

Page S10

(2,5-Dimethoxy-3-((4-methoxybenzyloxy)methyl)-phenyl]-methanol (29).

Page S11

X-Ray crystal structure of imide 19b.

Page S11

References

Page S12 
General Procedures. Unless otherwise stated, reaction solvents were dried by distillation under $\mathrm{N}_{2}$ from $\mathrm{CaH}_{2}\left(\mathrm{CH}_{2} \mathrm{Cl}_{2}, \mathrm{PhMe}\right.$, pyridine), sodium benzophenone ketyl $\left(\mathrm{Et}_{2} \mathrm{O}, \mathrm{THF}\right), \mathrm{K}_{2} \mathrm{CO}_{3}(\mathrm{MeOH})$ or obtained commercially anhydrous (DMF, $\mathrm{MeCN}, \mathrm{Et}_{3} \mathrm{~N}$, iso- $\mathrm{Pr}_{2} \mathrm{NEt}$, EtOH). Other solvents and all reagents were obtained from commercial suppliers and used without further purification unless otherwise stated. Reactions were performed in oven-dried glassware under $\mathrm{N}_{2}$ unless otherwise stated. Chromatographed refers to flash column chromatography on silica gel (eluants are given in parenthesis). Thin layer chromatography (TLC) was performed on pre-coated glass backed silica plates and visualized with a UV lamp (215 or $254 \mathrm{~nm})$, potassium permanganate or vanillin stains. Infrared spectra were recorded with automated background subtraction on thin films on sodium chloride plates. ${ }^{1} \mathrm{H}$ NMR spectra were recorded at 300 or $400 \mathrm{MHz}$ and ${ }^{13} \mathrm{C}$ NMR spectra at 75, 100 or $125 \mathrm{MHz}$. Chemical shifts ( $\delta)$ are quoted in parts per million ( $\mathrm{ppm})$ and are referenced to a residual solvent peak. Coupling constants $(J)$ are quoted in Hertz $(\mathrm{Hz})$ to the nearest $0.5 \mathrm{~Hz}$

1-Bromomethyl-2,5-dimethoxy-3-(methoxymethyl)-benzene (7a). $\mathrm{K}_{2} \mathrm{CO}_{3}(1.8 \mathrm{~g}, 13.2 \mathrm{mmol})$ and $\mathrm{Me}_{2} \mathrm{SO}_{4}(1.0 \mathrm{~mL}, 9.7 \mathrm{mmol})$ were successively added to phenol 28 (1.7 g, $\left.8.8 \mathrm{mmol}\right)$ in $\mathrm{Me}_{2} \mathrm{CO}$ (40 $\mathrm{mL})$. After $15 \mathrm{~h}, \mathrm{NH}_{3}$ and $\mathrm{MeOH}(1: 10,1 \mathrm{~mL})$ were added, the reaction mixture stirred for $1 \mathrm{~h}$, filtered through celite and rotary evaporated. The residue was dissolved in EtOAc $(100 \mathrm{~mL})$, washed once with saturated aqueous $\mathrm{NH}_{4} \mathrm{Cl}(10 \mathrm{~mL})$ and brine $(10 \mathrm{~mL})$, dried $\left(\mathrm{MgSO}_{4}\right)$ and again rotary evaporated. The residue was dissolved in THF $(16 \mathrm{~mL})$ and cooled to $0{ }^{\circ} \mathrm{C} . \mathrm{PPh}_{3}(1.8 \mathrm{~g}, 6.8 \mathrm{mmol})$ and NBS $(1.2 \mathrm{~g}$, $6.7 \mathrm{mmol}$ ) were successively added, the mixture stirred for $20 \mathrm{~min}$ at $0{ }^{\circ} \mathrm{C}$, diluted with pentane $(40 \mathrm{~mL})$, filtered through celite, rotary evaporated and chromatographed (pentane:EtOAc 5:1, 1\% $\left.\mathrm{Et}_{3} \mathrm{~N}\right)$ to obtain bromide $7 \mathbf{a}\left(1.27 \mathrm{~g}, 76 \%\right.$ ) as a colorless oil: $R_{f} 0.40$ (hexanes:EtOAc 7:1); IR (film) 1607, $1481,1430,1232,1194,1096,1054,951,859 \mathrm{~cm}^{-1} ;{ }^{1} \mathrm{H}$ NMR $\left(\mathrm{CDCl}_{3}, 300 \mathrm{MHz}\right) \delta 3.43(\mathrm{~s}, 3 \mathrm{H}), 3.79(\mathrm{~s}$, $3 \mathrm{H}), 3.85(\mathrm{~s}, 3 \mathrm{H}), 4.48(\mathrm{~s}, 2 \mathrm{H}), 4.54(\mathrm{~s}, 2 \mathrm{H}), 6.86(\mathrm{~d}, J=2.6 \mathrm{~Hz}, 1 \mathrm{H}), 6.93(\mathrm{~d}, J=2.6 \mathrm{~Hz}, 1 \mathrm{H}) ;{ }^{13} \mathrm{C} \mathrm{NMR}$ $\left(\mathrm{CDCl}_{3}, 75.5 \mathrm{MHz}\right) \delta 27.9,55.6,58.4,62.4,69.3,115.5,115.6,131.9,132.9,150.0,155.9 ; \mathrm{MS}$ (CI, 
$\left.\mathrm{NH}_{3}\right) \mathrm{m} / z$ 294, $292\left[\mathrm{M}+\mathrm{NH}_{4}^{+}\right]$; HRMS (CI) $m / z$ calc for $\mathrm{C}_{11} \mathrm{H}_{19} \mathrm{BrNO}_{3}:\left[\mathrm{M}+\mathrm{NH}_{4}\right]^{+}, 292.0548$; found:

$\left[\mathrm{M}+\mathrm{NH}_{4}\right]^{+}, 292.0545$

\section{1-Bromomethyl-2,5-dimethoxy-3-((4-methoxybenzyloxy)methyl)-benzene $\quad$ (7b). $\quad \mathrm{PPh}_{3} \quad(7.80 \mathrm{~g}$,}

$29.7 \mathrm{mmol})$ and $\mathrm{CBr}_{4}(10.4 \mathrm{~g}, 31.4 \mathrm{mmol})$ were successively added with stirring to alcohol 29 (5.26 g, $16.5 \mathrm{mmol})$ in $\mathrm{THF}(85 \mathrm{~mL})$ at $0{ }^{\circ} \mathrm{C}$. After $5 \mathrm{~min}$ at $0{ }^{\circ} \mathrm{C}$ followed by $70 \mathrm{~min}$ at room temperature, $\mathrm{Et}_{2} \mathrm{O}$ $(60 \mathrm{~mL})$ was added, the reaction mixture filtered through celite and rotary evaporated. This procedure was repeated three times. Finally $\mathrm{Et}_{2} \mathrm{O}$ and pentane $(3: 1,100 \mathrm{~mL})$ were added, the mixture filtered, rotary evaporated and chromatographed (pentane:EtOAc 10:1, 1\% $\left.\mathrm{Et}_{3} \mathrm{~N}\right)$ to give bromide $\mathbf{7 b}(4.58 \mathrm{~g}$, $73 \%$ ) as yellow oil: $R_{f} 0.33$ (hexanes:EtOAc 5:1); IR (film) 1611, 1513, 1480, 1321, 1246, 1173, 1055 $\mathrm{cm}^{-1} ;{ }^{1} \mathrm{H}$ NMR $\left(\mathrm{CDCl}_{3}, 300 \mathrm{MHz}\right) \delta 3.79(\mathrm{~s}, 3 \mathrm{H}), 3.81(\mathrm{~s}, 6 \mathrm{H}), 4.53(\mathrm{~s}, 2 \mathrm{H}), 4.54(\mathrm{~s}, 4 \mathrm{H}), 6.86(\mathrm{~d}, J=$ $3.0 \mathrm{~Hz}, 1 \mathrm{H}), 6.89(\mathrm{~d}, J=8.6 \mathrm{~Hz}, 2 \mathrm{H}), 6.97(\mathrm{~d}, J=2.6 \mathrm{~Hz}, 1 \mathrm{H}), 7.30(\mathrm{~d}, J=8.3 \mathrm{~Hz}, 2 \mathrm{H}) ;{ }^{13} \mathrm{C}$ NMR $\left(\mathrm{CDCl}_{3}, 75.5 \mathrm{MHz}\right) \delta 28.0,55.2,55.6,62.4,66.5,72.2,113.8,115.4,115.8,129.4,130.1,131.9,133.0$ 150.1, 155.8, 159.2; MS (CI, $\left.\mathrm{NH}_{3}\right) \mathrm{m} / z, 398\left[\mathrm{M}+\mathrm{NH}_{4}\right]^{+} ;$HRMS (CI) $m / z$ calc for $\mathrm{C}_{18} \mathrm{H}_{25} \mathrm{BrNO}_{4}:[\mathrm{M}+$ $\left.\mathrm{NH}_{4}\right]^{+}, 398.0967$; found: $\left[\mathrm{M}+\mathrm{NH}_{4}\right]^{+}, 398.0952$.

Methyl 2-hydroxy-4,6-dimethoxybenzoate (10). ${ }^{1} \mathrm{~K}_{2} \mathrm{CO}_{3}(265 \mathrm{~g}, 1.92 \mathrm{~mol})$ and $\mathrm{Me}_{2} \mathrm{SO}_{4}(180 \mathrm{~mL}$, $1.92 \mathrm{~mol})$ were added to 2,4,6-trihydroxybenzoic acid (9) $(60 \mathrm{~g}, 0.32 \mathrm{~mol})$ in $\mathrm{Me}_{2} \mathrm{CO}(1 \mathrm{~L})$ and the suspension was cooled to $0{ }^{\circ} \mathrm{C}$. After $2 \mathrm{~h}$ at $0{ }^{\circ} \mathrm{C}$, the ice bath was removed and stirring continued for $21 \mathrm{~h}$ at room temperature. The reaction was quenched by careful addition of a mixture of $\mathrm{MeOH}(75$ $\mathrm{mL})$ and saturated aqueous $\mathrm{NH}_{3}(25 \mathrm{~mL})$. After $1 \mathrm{~h}$, the reaction mixture was filtered into saturated aqueous $\mathrm{NH}_{3}$ and saturated aqueous $\mathrm{NH}_{4} \mathrm{Cl}(1: 4,250 \mathrm{~mL})$, stirring continued for $30 \mathrm{~min}$, the solution filtered again and the filtrate rotary evaporated. The aqueous layer was extracted with $\mathrm{Et}_{2} \mathrm{O}(1 \times$ $400 \mathrm{~mL}, 3 \times 100 \mathrm{~mL})$, the combined organic layers washed with brine $(200 \mathrm{~mL})$, dried $\left(\mathrm{MgSO}_{4}\right)$ and rotary evaporated. The dark brown residue oil was crystallized (150 mL, cyclohexane:EtOAc 6:1) to furnish white crystals $(61 \mathrm{~g}, 84 \%)$ of the corresponding ester. At $-78{ }^{\circ} \mathrm{C}, \mathrm{BCl}_{3}(190 \mathrm{~mL}, 1.0 \mathrm{M}$ in $\left.\mathrm{CH}_{2} \mathrm{Cl}_{2}\right)$ was added drop wise to the ester $(38.3 \mathrm{~g}, 172 \mathrm{mmol})$ in dry $\mathrm{CH}_{2} \mathrm{Cl}_{2}(750 \mathrm{~mL})$ under $\mathrm{N}_{2}$. After $1 \mathrm{~h}$, stirring was continued overnight at room temperature, when $10 \%$ aqueous $\mathrm{HCl}(450 \mathrm{~mL})$ was added. The aqueous layer was extracted with $\mathrm{CH}_{2} \mathrm{Cl}_{2}(3 \times 150 \mathrm{~mL})$, the combined organic layers were washed with saturated aqueous $\mathrm{NaHCO}_{3}(300 \mathrm{~mL})$ and brine $(200 \mathrm{~mL})$, dried $\left(\mathrm{MgSO}_{4}\right)$, rotary 
evaporated and recrystallized (cyclohexane:EtOAc 20:1) to give phenol $10(33.2 \mathrm{~g}, 91 \%)$ as a white solid: ${ }^{1} \mathrm{H}$ NMR $\left(\mathrm{CDCl}_{3}, 300 \mathrm{MHz}\right) \delta 3.82(\mathrm{~s}, 3 \mathrm{H}), 3.84(\mathrm{~s}, 3 \mathrm{H}), 3.93(\mathrm{~s}, 3 \mathrm{H}),, 5.98(\mathrm{~d}, 1 \mathrm{H}, J=2.2 \mathrm{~Hz}$,), $6.13(\mathrm{~d}, 1 \mathrm{H}, J=2.2 \mathrm{~Hz}), 12.06(\mathrm{~s}, 1 \mathrm{H}) ;{ }^{13} \mathrm{C} \mathrm{NMR}\left(\mathrm{CDCl}_{3}, 75 \mathrm{MHz}\right) \delta 52.2,55.5,56.1,91.6,93.4,96.6$, 162.2, 165.4, 166.0, 171.7; MS $\left(\mathrm{Cl}, \mathrm{NH}_{3}\right) \mathrm{m} / \mathrm{z} 213(\mathrm{M}+\mathrm{H})^{+}$; HRMS calc for $\mathrm{C}_{10} \mathrm{H}_{13} \mathrm{O}_{5}$ : $(\mathrm{M}+\mathrm{H})^{+}$, 213.0762; found: $(\mathrm{M}+\mathrm{H})^{+}, 213.0763$. The NMR spectroscopic data were consistent with those reported in literature. ${ }^{1}$

Methyl 3-formyl-2-hydroxy-4,6-dimethoxybenzoate (11). ${ }^{2} \mathrm{POCl}_{3}(16 \mathrm{~mL}, 170 \mathrm{mmol})$ was added to DMF $(33 \mathrm{~mL}, 0.42 \mathrm{~mol})$ at $0{ }^{\circ} \mathrm{C}$. After $1 \mathrm{~h}$, the reaction mixture was diluted with $\mathrm{MeCN}(50 \mathrm{~mL})$ and phenol 10 (18.0 g, $84.8 \mathrm{mmol})$ added portion wise. After $15 \mathrm{~min}$, the mixture was stirred over night at room temperature. The solution was cooled to $0{ }^{\circ} \mathrm{C}$, cold $\mathrm{H}_{2} \mathrm{O}(100 \mathrm{~mL})$ was added and the reaction mixture brought to $\mathrm{pH} 5-6$ by addition of $5 \mathrm{M} \mathrm{NaOH}(\sim 150 \mathrm{~mL})$. The aqueous layer was extracted with EtOAc $(1 \times 200 \mathrm{~mL}, 3 \times 100 \mathrm{~mL})$, the combined organic layers washed with brine $(2 \times 100 \mathrm{~mL})$, dried $\left(\mathrm{MgSO}_{4}\right)$, rotary evaporated and crystallized (EtOAc) to give aldehyde $11(14.3 \mathrm{~g}, 70 \%)$ as a white solid. The mother liquid was rotary evaporated and chromatographed $\left(100 \mathrm{~g} \mathrm{SiO}_{2}\right.$, $\mathrm{CH}_{2} \mathrm{Cl}_{2}$ to $\left.\mathrm{CH}_{2} \mathrm{Cl}_{2}: \mathrm{MeOH} 100: 1\right)$ to give phenol 10 (3.0 g, 17\%) and additional aldehyde 11 (1.0 g, 5\%): IR (film) 3438, 1725, 1632, 1476, 1277, 1217, 1126, $1107 \mathrm{~cm}^{-1} ;{ }^{1} \mathrm{H}$ NMR $\left(\mathrm{CDCl}_{3}, 300 \mathrm{MHz}\right) \delta 3.90(\mathrm{~s}$, $3 \mathrm{H}), 3.92(\mathrm{~s}, 3 \mathrm{H}), 3.93(\mathrm{~s}, 3 \mathrm{H}), 5.94(\mathrm{~s}, 1 \mathrm{H}), 10.11(\mathrm{~s}, 1 \mathrm{H}), 12.74(\mathrm{~s}, 1 \mathrm{H}) ;{ }^{13} \mathrm{C} \mathrm{NMR}\left(\mathrm{CDCl}_{3}, 75 \mathrm{MHz}\right) \delta$ 52.4, 56.0, 56.3, 85.9, 105.5, 162.9, 165.3, 192.0; $\mathrm{MS}\left(\mathrm{Cl}, \mathrm{NH}_{3}\right) \mathrm{m} / z 241(\mathrm{M}+\mathrm{H})^{+}$; HRMS calc for $\mathrm{C}_{11} \mathrm{H}_{13} \mathrm{O}_{6}:(\mathrm{M}+\mathrm{H})^{+}, 241.0703$; found: $(\mathrm{M}+\mathrm{H})^{+}, 241.0712$. The spectroscopic data for aldehyde 11 were consistent with those reported in literature. ${ }^{2}$

Dimethyl 5-chloro-2-hydroxy-4,6-dimethoxy-1,3-benzenedicarboxylate (16). When the oxidation of 11 was performed at $0{ }^{\circ} \mathrm{C}$ without 2-methylbutene, 16 was obtained (37\%) as a colorless oil: $R_{f} 0.32$ (hexanes:EtOAc 3:1); IR (film) 3452, 1738, 1662, $1607 \mathrm{~cm}^{-1} ;{ }^{1} \mathrm{H}$ NMR $\left(\mathrm{CDCl}_{3}, 300 \mathrm{MHz}\right) \delta 3.90(\mathrm{~s}$, 3H), $3.96(\mathrm{~s}, 3 \mathrm{H}), 11.81(\mathrm{~s}, 1 \mathrm{H}) ;{ }^{13} \mathrm{C} \mathrm{NMR}\left(\mathrm{CDCl}_{3}, 75 \mathrm{MHz}\right) \delta 53.0,61.8,109.8,114.3,159.3,159.4$, 167.4; $\mathrm{MS}\left(\mathrm{CI}, \mathrm{NH}_{3}\right) \mathrm{m} / z 336\left[\mathrm{M}+\mathrm{NH}_{4}\right]^{+}$; HRMS (CI) $m / z$ calc for $\mathrm{C}_{12} \mathrm{H}_{14} \mathrm{O}_{7} \mathrm{Cl}:[\mathrm{M}+\mathrm{H}]^{+}, 305.0428$; found: $[\mathrm{M}+\mathrm{H}]^{+}, 305.0441$.

Methyl 5,7-dimethoxy-1,3-dioxo-2,3-dihydro-1H-isoindole-4-carboxylate (17). During the preparation of 14, small amounts of imide 17 were obtained as a colorless oil: IR (film) 3418, 1768, 1724, 1626, 1433, 1387, 1325, $1284 \mathrm{~cm}^{-1} ;{ }^{1} \mathrm{H}$ NMR $\left(\mathrm{CDCl}_{3}, 300 \mathrm{MHz}\right) \delta 3.96(\mathrm{~s}, 6 \mathrm{H}), 4.04(\mathrm{~S}, 3 \mathrm{H}), 6.61$ 
(s, 1H), $7.77(\mathrm{~s}, 1 \mathrm{H}) ; \mathrm{MS}\left(\mathrm{CI}, \mathrm{NH}_{3}\right) \mathrm{m} / z 283\left[\mathrm{M}+\mathrm{NH}_{4}\right]^{+}, 266[\mathrm{M}+\mathrm{H}]^{+}, 102,85,52 ; \mathrm{HRMS}(\mathrm{CI}) \mathrm{m} / z$

calc for $\mathrm{C}_{12} \mathrm{H}_{12} \mathrm{NO}_{6}:[\mathrm{M}+\mathrm{H}]^{+}, 266.0665$; found: $[\mathrm{M}+\mathrm{H}]^{+}, 266.0665$.

Methyl 3-ethoxy-5,7-dimethoxy-1-oxo-2,3-dihydro-1H-isoindole-4-carboxylate (18). During the preparation of lactam 14, small amounts of lactam 18 were obtained as a colorless oil, when the reaction

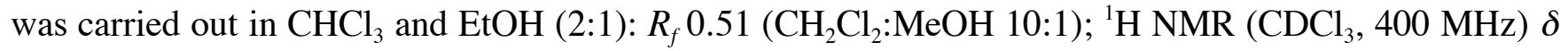
$1.10(\mathrm{t}, J=7.1 \mathrm{~Hz}, 3 \mathrm{H}), 3.26(\mathrm{dd}, J=15.9,7.1 \mathrm{~Hz}, 1 \mathrm{H}), 3.48(\mathrm{dd}, J=15.7,7.0 \mathrm{~Hz}, 1 \mathrm{H}), 3.88(\mathrm{~s}, 3 \mathrm{H})$, $3.94(\mathrm{~s}, 3 \mathrm{H}), 4.00(\mathrm{~s}, 3 \mathrm{H}), 6.07(\mathrm{~d}, J=1.3 \mathrm{~Hz}, 1 \mathrm{H}), 6.47(\mathrm{~s}, 1 \mathrm{H}), 6.88(\mathrm{bs}, 1 \mathrm{H}) ;{ }^{13} \mathrm{C} \mathrm{NMR}\left(\mathrm{CDCl}_{3}, 100.6\right.$ MHz) $\delta 15.1,52.2,56.2,56.5,82.7,96.1,110.8,111.3,147.4,160.1,163.2,165.2,168.2 ; \mathrm{MS}\left(\mathrm{CI}, \mathrm{NH}_{3}\right)$ $m / z, 296[\mathrm{M}+\mathrm{H}]^{+}, 336\left[\mathrm{M}+\mathrm{NH}_{4}\right]^{+} ; \operatorname{HRMS}(\mathrm{CI}) m / z$ calc for $\mathrm{C}_{14} \mathrm{H}_{18} \mathrm{NO}_{6}:[\mathrm{M}+\mathrm{H}]^{+}, 296.1134$; found: [M $+\mathrm{H}]^{+}, 296.1146$.

Methyl 5,7-dimethoxy-2-methyl-1,3-dioxo-2,3-dihydro-1H-isoindole-4-carboxylate (19a). When the methylation of lactam 14 was carried out without degassing, significant amounts $(\leq 25 \%)$ of imide 19a were obtained as a white solid: $R_{f} 0.20$ (EtOAc:hexanes 10:1); IR (film) 1765, 1711, 1628, 1601, 1504, 1438, 1378, 1324, $1283 \mathrm{~cm}^{-1}{ }_{;}^{1} \mathrm{H}$ NMR $\left(\mathrm{CDCl}_{3}, 300 \mathrm{MHz}\right) \delta 3.08(\mathrm{~s}, 3 \mathrm{H}), 3.94(\mathrm{~s}, 3 \mathrm{H}), 3.96(\mathrm{~s}, 3 \mathrm{H}), 4.03$ $(\mathrm{s}, 3 \mathrm{H}), 6.58(\mathrm{~s}, 1 \mathrm{H}) ;{ }^{13} \mathrm{C} \mathrm{NMR}\left(\mathrm{CDCl}_{3}, 75.5 \mathrm{MHz},\right) \delta 166.0,165.8,164.9,162.4,158.4,132.2,112.9$ 109.0, 99.2, 56.5, 56.4, 52.8, 23.6; MS $\left(\mathrm{CI}, \mathrm{NH}_{3}\right) m / z, 280[\mathrm{M}+\mathrm{H}]^{+}, 336\left[\mathrm{M}+\mathrm{NH}_{4}\right]^{+} ; \mathrm{HRMS}(\mathrm{CI}) m / z$ calc for $\mathrm{C}_{13} \mathrm{H}_{14} \mathrm{NO}_{6}:[\mathrm{M}+\mathrm{H}]^{+}, 280.0821$; found: $[\mathrm{M}+\mathrm{H}]^{+}, 280.0813$.

Methyl 7-methoxy-2-methyl-1,3-dioxo-5-trifluoromethanesulfonyloxy-2,3-dihydro-1H-isoindole-4carboxylate (19b): $\mathrm{BCl}_{3}\left(95 \mathrm{~mL}, 95 \mathrm{mmol}, 1.0 \mathrm{M}\right.$ in $\mathrm{CH}_{2} \mathrm{Cl}_{2}$ ) was added drop wise with stirring under $\mathrm{N}_{2}$ to amide $15(11.5 \mathrm{~g}, 43.3 \mathrm{mmol})$ so that the inside temperature remained below $-72{ }^{\circ} \mathrm{C}$. After $3 \mathrm{~h}$ at $-78{ }^{\circ} \mathrm{C}, 10 \%$ aqueous $\mathrm{HCl}(150 \mathrm{~mL})$ was added and the mixture allowed to warm up to room temperature and transferred to $10 \%$ aqueous $\mathrm{HCl}(1.3 \mathrm{~L})$ and $\mathrm{CH}_{2} \mathrm{Cl}_{2}(500 \mathrm{~mL})$. After stirring for $2 \mathrm{~h}$, the aqueous layer was extracted with $\mathrm{CH}_{2} \mathrm{Cl}_{2}$ and $\mathrm{CHCl}_{3}(3 \times 250 \mathrm{~mL})$ and $\mathrm{CHCl}_{3}$ and $i \operatorname{PrOH}(5: 1,2 \times$ $250 \mathrm{~mL})$, the combined organic layers washed with brine $(300 \mathrm{~mL})$, dried $\left(\mathrm{MgSO}_{4}\right)$, rotary evaporated 
and crystallized to give the corresponding phenol $(8.9 \mathrm{~g}, 82 \%)$ as a white solid: $\mathrm{mp} 198-203{ }^{\circ} \mathrm{C}$ (pentane); $R_{f} 0.44\left(\mathrm{CH}_{2} \mathrm{Cl}_{2}: \mathrm{MeOH} 15: 1\right)$; IR (film) 1713, 1672, 1596, 1467, 1431, 1336, 1271, 1219 $\mathrm{cm}^{-1} ;{ }^{1} \mathrm{H}$ NMR $\left(\mathrm{CDCl}_{3}, 300 \mathrm{MHz}\right) \delta 1.42(\mathrm{~s}, 1 \mathrm{H}), 3.14(\mathrm{~s}, 3 \mathrm{H}), 3.97(\mathrm{~s}, 3 \mathrm{H}), 4.00(\mathrm{~s}, 3 \mathrm{H}), 4.47(\mathrm{~s}, 2 \mathrm{H})$, $6.47(\mathrm{~s}, 1 \mathrm{H}) ;{ }^{13} \mathrm{C} \mathrm{NMR}\left(\mathrm{CDCl}_{3}, 75 \mathrm{MHz}\right) \delta 29.0,52.3,53.4,56.2,99.3,100.9,114.0,147.4,162.4$, 166.4, 167.2, 170.0; MS $\left(\mathrm{CI}, \mathrm{NH}_{3}\right) \mathrm{m} / z, 252[\mathrm{M}+\mathrm{H}]^{+}$; HRMS (CI) $m / z$ calc for $\mathrm{C}_{12} \mathrm{H}_{14} \mathrm{NO}_{5}:[\mathrm{M}+\mathrm{H}]^{+}$, 252.0872; found: $[\mathrm{M}+\mathrm{H}]^{+}, 252.0871$. Pyridine $(0.05 \mathrm{~mL}, 0.61 \mathrm{mmol})$ and $\mathrm{Tf}_{2} \mathrm{O}(0.09 \mathrm{~mL}, 0.53 \mathrm{mmol})$ were added successively with stirring to the phenol $(0.103 \mathrm{~g}, 0.4 \mathrm{mmol})$ in $\mathrm{CH}_{2} \mathrm{Cl}_{2}(5 \mathrm{~mL})$ at $0{ }^{\circ} \mathrm{C}$. After $4 \mathrm{~h}, \mathrm{H}_{2} \mathrm{O}$ was added and the separated organic phase dried $\left(\mathrm{MgSO}_{4}\right)$, filtered and rotary evaporated. Chromatography $\left(\mathrm{CH}_{2} \mathrm{Cl}_{2}\right)$ gave imide $19 \mathbf{b}\left(\mathrm{CH}_{2} \mathrm{Cl}_{2}\right.$, hexane, $\left.37 \%\right)$ as a colorless crystal: mp $132-134{ }^{\circ} \mathrm{C}$ $\left(\mathrm{CH}_{2} \mathrm{Cl}_{2}\right.$ :pentane); IR (film) 1775, 1745, 1628, 1498, 1431, 1381, 1304, $1210 \mathrm{~cm}^{-1} ;{ }^{1} \mathrm{H} \mathrm{NMR}\left(\mathrm{CDCl}_{3}\right.$, $300 \mathrm{MHz}) \delta 3.16(\mathrm{~s}, 3 \mathrm{H}), 4.02(\mathrm{~s}, 3 \mathrm{H}), 4.08(\mathrm{~s}, 3 \mathrm{H}), 7.14(\mathrm{~s}, 1 \mathrm{H}) ;{ }^{13} \mathrm{C} \mathrm{NMR}\left(\mathrm{CDCl}_{3}, 75 \mathrm{MHz}\right) \delta 24.2$, $53.4,57.2,110.8,116.4,117.2(\mathrm{q}, J=362.9 \mathrm{~Hz}), 120.6,133.4,150.6,154.6,157.9,161.8,164.5 ; \mathrm{MS}$ $\left(\mathrm{CI}, \mathrm{NH}_{3}\right) m / z 398[\mathrm{M}+\mathrm{H}]^{+} ; \mathrm{HRMS} m / z$ calc for $\mathrm{C}_{13} \mathrm{H}_{11} \mathrm{~F}_{3} \mathrm{NSO}_{8}:[\mathrm{M}+\mathrm{H}]^{+}$, 398.0157, found 398.0156.

\section{Methyl} 5-(2,5-dimethoxybenzyl)-7-methoxy-2-methyl-1-oxo-2,3-dihydro-1H-isoindole-4-

carboxylate (21). Benzylzinc chloride 20 in THF $(2.0 \mathrm{M} ; 6.2 \mathrm{~mL}, 3.1 \mathrm{mmol})$ was added at room temperature to a mixture of triflate $\mathbf{8}(535 \mathrm{mg}, 1.4 \mathrm{mmol})$ and $\mathrm{Pd}\left(\mathrm{PPh}_{3}\right)_{4}(81 \mathrm{mg}, 0.07 \mathrm{mmol})$ in $\mathrm{THF}(25$ $\mathrm{mL}$ ), which was degassed previously four times by a freeze-thaw process. The mixture was heated to reflux for $70 \mathrm{~min}$, cooled to room temperature and saturated aqueous $\mathrm{NH}_{4} \mathrm{Cl}(5 \mathrm{~mL})$ and $\mathrm{H}_{2} \mathrm{O}(10 \mathrm{~mL})$ were added and the aqueous layer extracted with EtOAc $(4 \times 50 \mathrm{~mL})$. The combined organic layers were washed with brine $(50 \mathrm{~mL})$, dried $\left(\mathrm{MgSO}_{4}\right)$, rotary evaporated and chromatographed (EtOAc to EtOAc:MeOH 30:1) to yield ester 21 (510 mg, 94\%) as a white oil: $R_{f} 0.31$ (EtOAc:MeOH 20:1); IR (film) 1712, 1682, 1594, 1499, 1433, 1277, 1226, 1159, $10521021 \mathrm{~cm}^{-1} ;{ }^{1} \mathrm{H} \mathrm{NMR}\left(\mathrm{CDCl}_{3}, 300 \mathrm{MHz}\right) \delta$ $3.15(\mathrm{~s}, 3 \mathrm{H}), 3.71(\mathrm{~s}, 3 \mathrm{H}), 3.78(\mathrm{~s}, 3 \mathrm{H}), 3.85(\mathrm{~s}, 3 \mathrm{H}), 3.88(\mathrm{~s}, 3 \mathrm{H}), 4.39(\mathrm{~s}, 2 \mathrm{H}), 4.55(\mathrm{~s}, 2 \mathrm{H}), 6.52-6,54$ $(\mathrm{m}, 1 \mathrm{H}), 6.73-6.82(\mathrm{~m}, 3 \mathrm{H}) ;{ }^{13} \mathrm{C} \mathrm{NMR}\left(\mathrm{CDCl}_{3}, 75 \mathrm{MHz}\right) \delta 29.1,51.6,53.4,55.6,55.9,111.1,111.2$, 113.6, 116.6, 117.5, 127.7, 130.1, 134.4, 146.8, 148.7, 151.5, 159.1, 166.7; MS (CI, $\left.\mathrm{NH}_{3}\right) m / z 386[\mathrm{M}+$ 
$\mathrm{H}]^{+}$; HRMS (CI) $m / z$ calc for $\mathrm{C}_{21} \mathrm{H}_{24} \mathrm{NO}_{6}:[\mathrm{M}+\mathrm{H}]^{+}, 386.1603$; found: $[\mathrm{M}+\mathrm{H}]^{+}, 386.1606$.

5-(2,5-Dimethoxybenzyl)-7-methoxy-2-methyl-1-oxo-2,3-dihydro-1H-isoindole-4-carboxylic acid (22). $\mathrm{LiOH} \cdot \mathrm{H}_{2} \mathrm{O}(284 \mathrm{mg}, 6.60 \mathrm{mmol})$ was added at room temperature to ester $21(510 \mathrm{mg}, 1.32 \mathrm{mmol})$ in THF $(10 \mathrm{~mL}), \mathrm{MeOH}(5 \mathrm{~mL})$ and $\mathrm{H}_{2} \mathrm{O}(5 \mathrm{ml})$. After stirring for $40 \mathrm{~h}$, saturated aqueous $\mathrm{NH}_{4} \mathrm{Cl}(5 \mathrm{~mL})$ and $\mathrm{H}_{2} \mathrm{O}(4 \mathrm{~mL})$ were added and the aqueous layer extracted with EtOAc $(2 \times 50 \mathrm{~mL})$ and $\mathrm{CHCl}_{3}$ and $i \mathrm{PrOH}(5: 1,2 \times 30 \mathrm{~mL})$. The combined organic layers were dried $\left(\mathrm{Na}_{2} \mathrm{SO}_{4}\right)$, rotary evaporated and chromatographed $\left(\mathrm{CH}_{2} \mathrm{Cl}_{2}: \mathrm{MeOH} 10: 1\right)$ to give recovered ester 21 (60 mg, 11\%) and acid 22 (180 mg, 37\%) as a white oil: $R_{f} 0.48\left(\mathrm{CH}_{2} \mathrm{Cl}_{2}: \mathrm{MeOH} 10: 1\right)$; IR (film) 1707, 1651, 1497, 1421, 1278, 1242, 1161, $1052 \mathrm{~cm}^{-1} ;{ }^{1} \mathrm{H}$ NMR $\left(\mathrm{CDCl}_{3}, 300 \mathrm{MHz}\right) \delta 3.13(\mathrm{~s}, 3 \mathrm{H}), 3.69(\mathrm{~s}, 3 \mathrm{H}), 3.73(\mathrm{~s}, 3 \mathrm{H}), 3.82$ $(\mathrm{s}, 3 \mathrm{H}), 4.46(\mathrm{~s}, 2 \mathrm{H}), 4.66(\mathrm{~s}, 2 \mathrm{H}), 6.57(\mathrm{~s}, 1 \mathrm{H}), 6.63-6.88(\mathrm{~m}, 3 \mathrm{H}) ;{ }^{13} \mathrm{C} \mathrm{NMR}\left(\mathrm{CDCl}_{3}, 75.5 \mathrm{MHz}\right) \delta$ $170.6,166.5,159.6,153.5,151.6,150.1,148.0,129.9,119.3,116.8,116.3,113.4,111.3,111.1,55.9$, 55.6, 53.8, 35.0, 29.1; MS (CI, $\left.\mathrm{NH}_{3}\right) \mathrm{m} / z 372[\mathrm{M}+\mathrm{H}]^{+}$; HRMS (CI) $m / z$ calc for $\mathrm{C}_{20} \mathrm{H}_{22} \mathrm{NO}_{6}:[\mathrm{M}+\mathrm{H}]^{+}$, 372.1447; found: $[\mathrm{M}+\mathrm{H}]^{+}, 372.1452$.

2-Methyl-4,7,10,11-tetramethoxy-1,2-dihydronaphtho[2,3-e]isoindol-3-one (24a). At $110{ }^{\circ} \mathrm{C}$ acid 22 (100 mg, $0.27 \mathrm{mmol}$ ) was added to polyphosphoric acid $(3 \mathrm{~mL})$. After $4.5 \mathrm{~h}$ stirring at $110{ }^{\circ} \mathrm{C}$, the deep red mixture was cooled to room temperature, $\mathrm{H}_{2} \mathrm{O}(5 \mathrm{~mL})$ was added, the reaction mixture brought to pH 4 with aqueous $\mathrm{NaOH}(5 \mathrm{~mL})$ and extracted with EtOAc $(3 \times 50 \mathrm{~mL})$. The combined organic layers were washed with brine $(20 \mathrm{~mL})$, dried $\left(\mathrm{MgSO}_{4}\right)$, rotary evaporated and redissolved in $\mathrm{Me}_{2} \mathrm{CO}(3 \mathrm{~mL})$. $\mathrm{K}_{2} \mathrm{CO}_{3}(40 \mathrm{mg}, 0.28 \mathrm{mmol})$ and $\mathrm{Me}_{2} \mathrm{SO}_{4}(30 \mu \mathrm{L}, 30 \mathrm{mmol})$ were added and, after $4 \mathrm{~h}$ at reflux, $\mathrm{H}_{2} \mathrm{O}$ was added. The reaction mixture was extracted with EtOAc $(3 \times 10 \mathrm{~mL})$, washed with brine $(2 \times 10 \mathrm{~mL})$, dried $\left(\mathrm{MgSO}_{4}\right)$, purified by chromatography on Florisil (EtOAc:MeOH 20:1) to give lactam $24 a(7 \mathrm{mg}$, 20\%) as a deep yellow oil: $R_{f} 0.43$ (EtOAc:MeOH 10:1); IR (film) 1682, 1615, 1459, 1424, 1358, 1322 , 1257, 1107, 1076, 1025, 975, $730 \mathrm{~cm}^{-1} ;{ }^{1} \mathrm{H}$ NMR $\left(\mathrm{CDCl}_{3}, 300 \mathrm{MHz}\right) \delta 3.26(\mathrm{~s}, 3 \mathrm{H}), 3.96(\mathrm{~s}, 3 \mathrm{H}), 4.02$ $(\mathrm{s}, 3 \mathrm{H}), 4.03(\mathrm{~s}, 3 \mathrm{H}), 4.05(\mathrm{~s}, 3 \mathrm{H}), 4.92(\mathrm{~s}, 2 \mathrm{H}), 6.66(\mathrm{~s}, 2 \mathrm{H}), 7.21(\mathrm{~s}, 1 \mathrm{H}), 8.53(\mathrm{~s}, 1 \mathrm{H}) ;{ }^{13} \mathrm{C} \mathrm{NMR}$ 
$\left(\mathrm{CDCl}_{3}, 75.5 \mathrm{MHz}\right) \delta 29.3,54.2,55.8,56.3,63.8,102.4,102.8,105.0,115.9,117.0,117.9,123.3,128.7$, 134.8, 142.8, 149.2, 149.6, 153.9, 154.4, 167.2; MS (CI, $\left.\mathrm{NH}_{3}\right) \mathrm{m} / \mathrm{z} 368[\mathrm{M}+\mathrm{H}]^{+} ; \mathrm{HRMS}(\mathrm{CI}) \mathrm{m} / z$ calc for $\mathrm{C}_{21} \mathrm{H}_{22} \mathrm{NO}_{5}:[\mathrm{M}+\mathrm{H}]^{+}, 368.1498$; found: $[\mathrm{M}+\mathrm{H}]^{+}, 368.1489$.

\section{2-Methyl-3-oxo-4,7,10-trimethoxy-2,3-dihydro-1H-naphtho[2,3-e]isoindol-11-yl acetate (24b).}

$\mathrm{Me}_{2} \mathrm{C}=\mathrm{C}(\mathrm{Cl}) \mathrm{NMe}_{2}(52 \mu \mathrm{L}, 0.39 \mathrm{mmol})$ was added drop wise to acid $22(35 \mathrm{mg}, 0.094 \mathrm{mmol})$ in dry $\mathrm{CH}_{2} \mathrm{Cl}_{2}(1.9 \mathrm{~mL})$. After $3 \mathrm{~h}$, the deep yellow mixture was cooled to $0{ }^{\circ} \mathrm{C}, \mathrm{ZnCl}_{2}$ in $\mathrm{Et}_{2} \mathrm{O}(1.0 \mathrm{M} ; 162 \mu \mathrm{L})$ was added and the mixture stirred for 40 min with a resultant color change to orange. Pyridine ( $3 \mathrm{~mL})$, $\mathrm{Ac}_{2} \mathrm{O}(1.00 \mathrm{~mL})$ and DMAP $(6 \mathrm{mg}, 0.050 \mathrm{mmol})$ were added and stirring continued for further $15 \mathrm{~h}$. $\mathrm{H}_{2} \mathrm{O}(2 \mathrm{~mL})$ and $\mathrm{HCl}(10 \%, 6 \mathrm{~mL})$ were added, the aqueous layer extracted with EtOAc $(5 \times 15 \mathrm{~mL})$, the combined organic layers washed with saturated aqueous $\mathrm{NaHCO}_{3}(2 \times 5 \mathrm{~mL})$, brine $(5 \mathrm{~mL})$, dried $\left(\mathrm{MgSO}_{4}\right)$ and rotary evaporated. The residue was chromatographed $\left(\mathrm{CHCl}_{3}\right)$ to yield acetate $\mathbf{2 4 b}(30 \mathrm{mg}$, $80 \%)$ as a deep yellow-greenish oil: $R_{f} 0.27\left(\mathrm{CHCl}_{3}\right)$; IR (film) $1769,1681 \mathrm{~cm}^{-1} ;{ }^{1} \mathrm{H}$ NMR $\left(\mathrm{CDCl}_{3}, 400\right.$ MHz) $\delta 2.47$ (s, 3H), 3.21 (s, 3H), $3.92(\mathrm{~s}, 3 \mathrm{H}), 3.99$ (s, 3H), 4.02 (s, 3H), 4.51 (bs, 1H), 4.78 (bs, 1H), $6.62(\mathrm{~s}, 2 \mathrm{H}), 7.15(\mathrm{~s}, 1 \mathrm{H}), 8.57(\mathrm{~s}, 1 \mathrm{H}) ;{ }^{13} \mathrm{C} \mathrm{NMR}\left(\mathrm{CDCl}_{3}, 100 \mathrm{MHz}\right) \delta 21.4,29.2,53.3,55.7,56.1$, 102.4, 103.5, 105.2, 116.5, 117.0, 118.2, 123.8, 127.7, 134.2, 141.3, 142.3, 148.7, 149.0, 154.1, 166.8, 169.5; MS (CI, $\left.\mathrm{NH}_{3}\right) \mathrm{m} / z 396[\mathrm{M}+\mathrm{H}]^{+}$; HRMS (CI) $m / z$ calc for $\mathrm{C}_{22} \mathrm{H}_{22} \mathrm{NO}_{6}:[\mathrm{M}+\mathrm{H}]^{+}, 368.1447$; found: $[\mathrm{M}+\mathrm{H}]^{+}, 368.1449$.

\section{4-Methoxy-2-methyl-3,7,10-trioxo-2,3,7,10-tetrahydro-1 $H$-naphtho[2,3-e]isoindol-11-yl acetate}

(25b). CAN (58 mg, $0.105 \mathrm{mmol})$ dissolved in $\mathrm{H}_{2} \mathrm{O}(0.5 \mathrm{~mL})$ was slowly added to acetate $\mathbf{2 4 b}(20 \mathrm{mg}$, $0.05 \mathrm{mmol})$ in $\mathrm{MeCN}(2 \mathrm{~mL})$ at $0{ }^{\circ} \mathrm{C}$. After $10 \mathrm{~min}$, the yellow mixture was stirred for $20 \mathrm{~h}$ at room temperature. $\mathrm{H}_{2} \mathrm{O}(3 \mathrm{~mL})$ was added, the mixture extracted with EtOAc $(4 \times 10 \mathrm{~mL})$, the combined organic layers washed with brine $(10 \mathrm{~mL})$, dried $\left(\mathrm{MgSO}_{4}\right)$, rotary evaporated and chromatographed $\left(\mathrm{CHCl}_{3}\right)$ to yield quinone $25 \mathrm{~b}(12 \mathrm{mg}, 65 \%)$ as a yellow/orange oil: $R_{f} 0.32\left(\mathrm{CHCl}_{3}\right)$; IR (film) 2359 , $2341,1777,1690,1673,1603,1426 \mathrm{~cm}^{-1} ;{ }^{1} \mathrm{H}$ NMR $\left(\mathrm{CDCl}_{3}, 400 \mathrm{MHz}\right) \delta 2.64(\mathrm{~s}, 3 \mathrm{H}), 3.26(\mathrm{~s}, 3 \mathrm{H}), 4.12$ 
(s, 3H), 4.63 (bs, 1H), $4.91(\mathrm{bs}, 1 \mathrm{H}), 6.93(\mathrm{~d}, 1 \mathrm{H}, J=10.3 \mathrm{~Hz}), 7.02(\mathrm{~d}, 1 \mathrm{H}, J=10.3 \mathrm{~Hz}), 7.37(\mathrm{~s}, 1 \mathrm{H})$,

$8.50(\mathrm{~s}, 1 \mathrm{H}) ;{ }^{13} \mathrm{C} \mathrm{NMR}\left(\mathrm{CDCl}_{3}, 100 \mathrm{MHz},\right) \delta 21.8,29.4,52.9,56.3,108.7,117.0,120.6,125.7,126.1$, $130.2,138.3,139.6,141.5,142.7,157.9,165.8,168.7,183.1,183.8 ; \mathrm{MS}\left(\mathrm{CI}, \mathrm{NH}_{3}\right) \mathrm{m} / z .366[\mathrm{M}+\mathrm{H}]^{+}$.

2-Hydroxymethyl-4-methoxy-6-(methoxymethyl)-phenol (28). pTsOH (2.0 g, $11 \mathrm{mmol})$ was added with stirring at room temperature to triol $27^{3}(22.4 \mathrm{~g}, 122 \mathrm{mmol})$ in $\mathrm{Me}_{2} \mathrm{CO}(100 \mathrm{~mL})$ and 2,2-dimethoxypropane $(200 \mathrm{~mL})$. After $1 \mathrm{~h}, \mathrm{NaHCO}_{3}(5 \mathrm{~g})$ was added and the solvent rotary evaporated. The residue was redissolved in EtOAc $(250 \mathrm{~mL})$ and washed twice with $\mathrm{H}_{2} \mathrm{O}(2 \times 60 \mathrm{~mL})$. The organic layer was rotary evaporated, $50 \%$ aqueous $\mathrm{AcOH}(20 \mathrm{~mL})$ added and the mixture stirred for $30 \mathrm{~min}$. The mixture was diluted with $\mathrm{Et}_{2} \mathrm{O}(300 \mathrm{~mL})$, the organic layer washed with $\mathrm{H}_{2} \mathrm{O}(60 \mathrm{~mL}), \mathrm{NaHCO}_{3}(2 \times$ $50 \mathrm{~mL})$ and brine $(80 \mathrm{~mL})$, dried $\left(\mathrm{MgSO}_{4}\right)$ and rotary evaporated to give the corresponding acetonide ${ }^{4}$ $(25.5 \mathrm{~g}, 93 \%)$ as a white solid. $\mathrm{NaH}(3.2 \mathrm{~g}, 79.7 \mathrm{mmol}, 60 \%$ in mineral oil) and MeI (4.9 mL, $79.7 \mathrm{mmol})$ were added subsequently at $0{ }^{\circ} \mathrm{C}$ to the crude acetonide (14.9 $\left.\mathrm{g}, 66.4 \mathrm{mmol}\right)$ in THF (120 $\mathrm{mL})$. After $3 \mathrm{~h}$, saturated aqueous $\mathrm{NH}_{3}$ and saturated aqueous $\mathrm{NH}_{4} \mathrm{Cl}(1: 4,20 \mathrm{~mL})$ were added, the mixture stirred for $1 \mathrm{~h}$ at room temperature and the aqueous layer extracted with EtOAc $(1 \times 100 \mathrm{~mL}, 2$ $\times 50 \mathrm{~mL})$. The combined organic layers were washed with brine $(100 \mathrm{~mL})$, dried $\left(\mathrm{MgSO}_{4}\right)$ and rotary evaporated. The residue was dissolved in THF $(120 \mathrm{~mL})$ and $\mathrm{H}_{2} \mathrm{O}(30 \mathrm{~mL})$ and cooled to $0{ }^{\circ} \mathrm{C}$. $p$ TsOH (18 $\mathrm{g}, 94 \mathrm{mmol}$ ) was added in portions, the ice bath removed and the mixture stirred over night and quenched by addition of saturated aqueous $\mathrm{NaHCO}_{3}(200 \mathrm{~mL})$ and $\mathrm{H}_{2} \mathrm{O}(50 \mathrm{~mL})$. The aqueous layer was extracted with EtOAc $(1 \times 300,2 \times 100 \mathrm{~mL})$, the combined organic layers washed with brine $(100 \mathrm{~mL})$, dried $\left(\mathrm{MgSO}_{4}\right)$, rotary evaporated to give phenol $28(13 \mathrm{~g}, 98 \%)$ as a viscous, brown oil: $R_{f} 0.24$ (EtOAc:hexane 1:1); IR (film) 3368, 1612, 1482, 1382, $1332 \mathrm{~cm}^{-1} ;{ }^{1} \mathrm{H} \mathrm{NMR}\left(\mathrm{CDCl}_{3}, 300 \mathrm{MHz},\right) \delta 2.62$ (bs, 1H), $3.45(\mathrm{~s}, 3 \mathrm{H}), 3.75(\mathrm{~s}, 3 \mathrm{H}), 4.60(\mathrm{~s}, 2 \mathrm{H}), 4.71(\mathrm{~s}, 2 \mathrm{H}), 6.59(\mathrm{~d}, J=2.6 \mathrm{~Hz}, 1 \mathrm{H}), 6.72(\mathrm{~d}, J=$ $2.6 \mathrm{~Hz}, 1 \mathrm{H}), 7.53(\mathrm{~s}, 1 \mathrm{H}) ;{ }^{13} \mathrm{C} \mathrm{NMR}\left(\mathrm{CDCl}_{3}, 75.5 \mathrm{MHZ}\right) \delta 55.6,58.2,62.4,72.3,113.0,113.1,123.7$, 127.5, 147.8, 152.5; MS (CI, $\left.\mathrm{NH}_{3}\right) m / z$ 198, $216\left[\mathrm{M}+\mathrm{NH}_{4}\right]^{+}$; HRMS (CI) $m / z$ calc for $\mathrm{C}_{10} \mathrm{H}_{18} \mathrm{NO}_{4}:[\mathrm{M}+$ 
$\left.\mathrm{NH}_{4}\right]^{+}, 216.1236$; found: $\left[\mathrm{M}+\mathrm{NH}_{4}\right]^{+}, 216.1237$.

(2,5-Dimethoxy-3-((4-methoxybenzyloxy)methyl)-phenyl)-methanol (29). $\mathrm{K}_{2} \mathrm{CO}_{3}(17.6 \mathrm{~g}, 127 \mathrm{mmol})$ and $\mathrm{Me}_{2} \mathrm{SO}_{4}(11.1 \mathrm{~mL}, 117 \mathrm{mmol})$ were added at room temperature to triol $27(19.6 \mathrm{~g}, 106 \mathrm{mmol})$ in $\mathrm{Me}_{2} \mathrm{CO}(600 \mathrm{~mL})$. After stirring at reflux for $4 \mathrm{~h}, \mathrm{MeOH}(50 \mathrm{~mL})$ and saturated aqueous $\mathrm{NH}_{3}(20 \mathrm{~mL})$ were added, the precipitate was filtered off, washed with EtOAc and the filtrate rotary evaporated. The residue was dissolved in EtOAc $(300 \mathrm{~mL})$ and extracted with saturated aqueous $\mathrm{NaHCO}_{3}(100 \mathrm{~mL})$ and $\mathrm{H}_{2} \mathrm{O}(50 \mathrm{~mL})$. The aqueous layer was washed with EtOAc $(3 \times 75 \mathrm{~mL})$ and the combined organic layers were washed with brine $(150 \mathrm{~mL})$, dried $\left(\mathrm{MgSO}_{4}\right)$ and rotary evaporated. The residue was crystallized from EtOAc and $\mathrm{Et}_{2} \mathrm{O}$ (2:1) to give (3-(hydroxymethyl)-2,5-dimethoxyphenyl)-methanol ${ }^{5}$ (14.8 g, 70\%) as a white solid. This was dissolved in THF and DMF $(5: 1,150 \mathrm{~mL})$ at $0{ }^{\circ} \mathrm{C}$ and $\mathrm{NaH}(1.45 \mathrm{~g}$, $36.0 \mathrm{mmol}, 60 \%$ in mineral oil) followed by $\mathrm{PMBCl}(4.9 \mathrm{~mL}, 36 \mathrm{mmol})$ were added. After $2 \mathrm{~h}$, the mixture was warmed up to room temperature and stirred for $100 \mathrm{~h}$. Saturated aqueous $\mathrm{NH}_{4} \mathrm{Cl}(50 \mathrm{~mL})$, $\mathrm{H}_{2} \mathrm{O}(50 \mathrm{~mL})$ and EtOAc $(200 \mathrm{~mL})$ were added and the aqueous layer extracted with EtOAc $(3 \times$ $100 \mathrm{~mL})$. The combined organic layers were washed with $\mathrm{H}_{2} \mathrm{O}(100 \mathrm{~mL})$, brine $(150 \mathrm{~mL})$, dried $\left(\mathrm{MgSO}_{4}\right)$, rotary evaporated and chromatographed (pentane:EtOAc 1:1 to 2:3) to yield PMB-ether 29 $(5.49 \mathrm{~g}, 43 \%)$ as a yellow oil, along with recovered starting material $(2.61 \mathrm{~g}, 33 \%): R_{f} 0.24$ (EtOAc:hexanes 1:1); IR (film) 3400 (bs), 1611, 1513, 1478, 1248, 1174, 1058, 1033, 1009, 851, 821 $\mathrm{cm}^{-1} ;{ }^{1} \mathrm{H} \mathrm{NMR}\left(\mathrm{CDCl}_{3,} 300 \mathrm{MHz}\right) \delta 1.78-2.46(\mathrm{bs}, 1 \mathrm{H}), 3.71(\mathrm{~s}, 3 \mathrm{H}), 3.78(\mathrm{~s}, 3 \mathrm{H}), 3.80(\mathrm{~s}, 3 \mathrm{H}), 4.53(\mathrm{~s}$, 2H), $4.68(\mathrm{~s}, 4 \mathrm{H}), 6.76-6.98(\mathrm{~m}, 4 \mathrm{H}), 7.19-7.36(\mathrm{~m}, 4 \mathrm{H}) ;{ }^{13} \mathrm{C} \mathrm{NMR}\left(\mathrm{CDCl}_{3}, 75.5 \mathrm{MHz}\right) \delta 55.2,55.5$, $61.0,62.3,66.5,72.2,113.6,113.7,114.1,129.4,130.1,132.4,134.8,149.8,156.0,159.2$; MS (CI, $\left.\mathrm{NH}_{3}\right) m / z 336\left[\mathrm{M}+\mathrm{NH}_{4}\right]^{+}$; HRMS (CI) $m / z$ calc for $\mathrm{C}_{18} \mathrm{H}_{26} \mathrm{NO}_{5}:\left[\mathrm{M}+\mathrm{NH}_{4}\right]^{+}, 336.1810$; found: $[\mathrm{M}+$ $\left.\mathrm{NH}_{4}\right]^{+}, 336.1803$.

\section{X-Ray crystal structure of imide 19b.}

Crystal data for 19b: $\mathrm{C}_{13} \mathrm{H}_{10} \mathrm{~F}_{3} \mathrm{NO}_{8} \mathrm{~S}, M=397.28$, monoclinic, $P 2_{1} / c$ (no. 14), $a=19.642(7), b=$ 
10.200(4), $c=8.221(3) \AA, \beta=95.40(2)^{\circ}, V=1639.8(11) \AA^{3}, Z=4, D_{c}=1.609 \mathrm{~g} \mathrm{~cm}^{-3}, \mu(\mathrm{Cu}-\mathrm{K \alpha} \alpha)=$ $2.499 \mathrm{~mm}^{-1}, T=293 \mathrm{~K}$, colorless plates; 2430 independent measured reflections, $F^{2}$ refinement, $R_{1}=$ $0.082, w R_{2}=0.208,1211$ independent observed absorption-corrected reflections $\left[\left|F_{\mathrm{o}}\right|>4 \sigma\left(\left|F_{\mathrm{o}}\right|\right), 2 \theta_{\max }=\right.$ $120^{\circ}$ ], 237 parameters. CCDC 612587.

Fig. S1 The molecular structure of $\mathbf{1 9 b}$ (20\% probability ellipsoids).

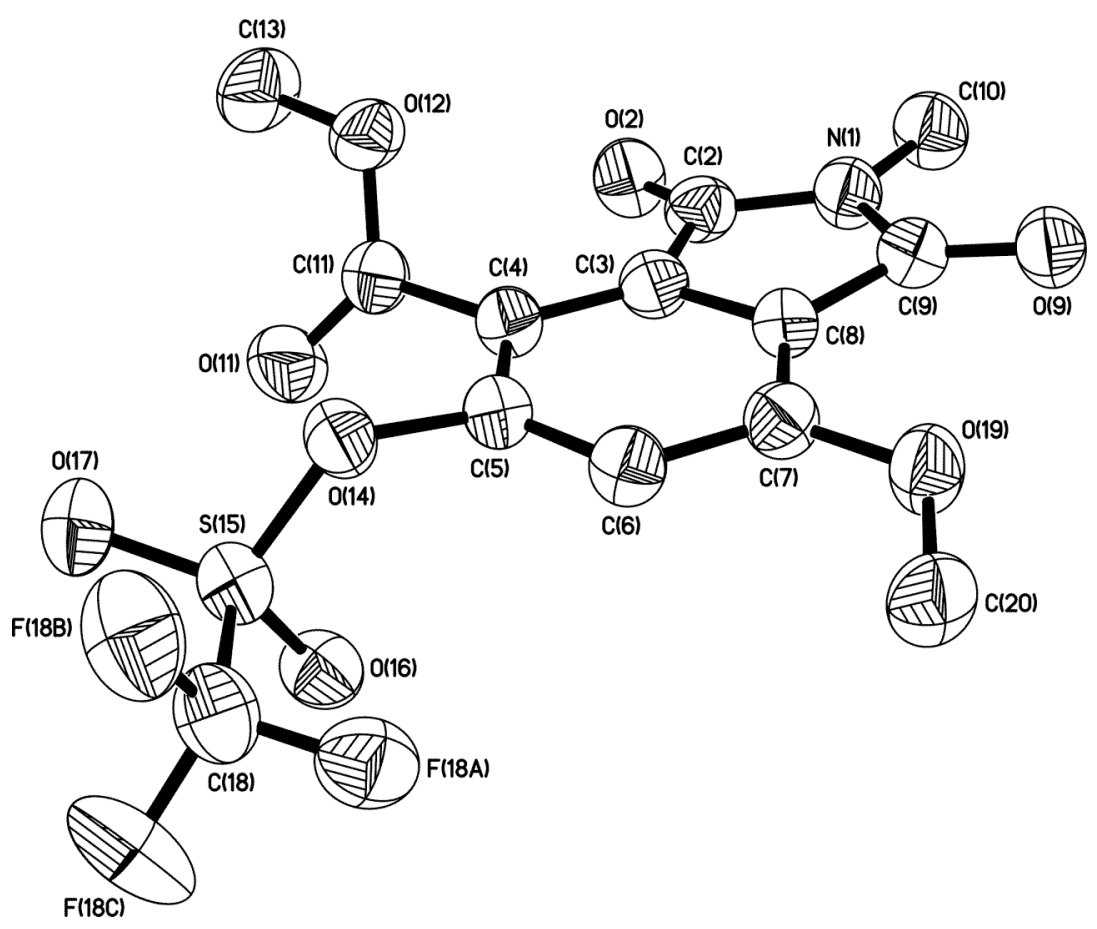

Fig. S1

\section{References}

(1) Rossi, R.; Carpita, A.; Bellina, F.; Stabile, P.; Mannina, L. Tetrahedron 2003, 59, 2067.

(2) Balgir, B. S.; Mander, L. N.; Mander, S. T. K. Aust. J. Chem. 1973, 26, 2459.

(3) Moran, W. J.; Schreiber, E. C.; Engel, E.; Behn, D. C.; Yasmins, J. L. J. Am. Chem. Soc. $1952,74,127$. 
(4) Clark, D. A.; Riccardis, F. De; Nicolaou, K. C. Tetrahedron 1994, 50, 11391.

(5) Witiak, D. T.; Kamat, P. L.; Allison, D. L.; Liebowitz, S. M.; Glaser, R.; Holliday, J. E.; Moeschberger, M. L.; Schaller, J. P. J. Med. Chem. 1983, 26, 1679. 